\title{
NiÑOS SOCIALMENTE hÁBILES: ¿CUÁNTO INFLUYEN LA EMPATía Y LAS EMOCIONES POSITIVAS?*
}

\section{SOCIALLY SKILLFUL CHILDREN: HOW MUCH INFLUENCE DO POSITIVE EMOTIONS AND EMPATHY HAVE?}

\author{
Laura B. Oros ${ }^{* *}$ y Andrea C. Fontana Nalesso***
}

\begin{abstract}
*Investigación financiada por el Instituto Superior Adventista de Misiones.
${ }^{* *}$ Doctora en Psicología. Miembro de la Carrera del Investigador Científico del Consejo Nacional de Investigaciones Científicas y Técnicas (CONICET). Investigadora del Instituto de Investigación Científica (IDIC) de la Universidad de la Cuenca del Plata (UCP). Directora del Departamento de Investigación del Instituto Superior Adventista de Misiones. E-Mail: lauraorosb@gmail.com *** Licenciada en Psicología. Docente del Instituto Superior Adventista de Misiones. Av. Rusia 410 - (N3315WAA) Leandro N. Alem. Misiones, Argentina. E-Mail: lauraorosb@gmail.com
\end{abstract}

\section{RESUMEN}

El análisis de los factores que propician el desarrollo de habilidades sociales en la niñez constituye un reto de gran relevancia para la psicología actual. El objetivo del estudio realizado fue analizar si la empatía y la experiencia de emociones positivas impactan significativamente sobre la ejecución de habilidades sociales en la niñez media, y en qué medida lo hace cada una. Se seleccionó una muestra no aleatoria de 406 niños (227 niñas y 179 varones), comprendidos mayormente entre los 10 y 12 años de edad, quienes completaron los siguientes instrumentos, previo consentimiento de sus padres: (a) la Subescala de Habilidades Sociales Adecuadas (Matson, Rotatori \& Helsel, 1983), validada a la Argentina por Schulz (2008), (b) la Escala Multidimensional de Empatía para niños argentinos (Richaud de Minzi, Lemos \& Oros, 2013) y (c) el Cuestionario Infantil de Emociones Positivas (Oros, 2014). Para analizar la acción conjunta de las emociones positivas y la empatía sobre las habilidades sociales apropiadas, se ejecutó un ANOVA factorial con un diseño 3 (alta, moderada y baja emocionalidad positiva) x 3 (alta, moderada y baja empatía). La variable dependiente estuvo constituida por los valores brutos de la Subescala de Habilidades Sociales Adecuadas. Los resultados indicaron que las emociones positivas y la empatía facilitan el despliegue de conductas socialmente habilidosas, siendo mayor el impacto de las emociones positivas en comparación a la empatía. Estos resultados son importantes a la hora de comprender la dinámica de los procesos implicados y de diseñar programas de intervención para promover habilidades sociales en la niñez.

Palabras clave: Habilidades sociales; Empatía; Emociones positivas; Niños.

\section{ABSTRACT}

The implementation of social skills, such as maintaining eye contact with another person during a conversation, giving and receiving compliments, helping others, sharing something, giving thanks, asking for permission, apologizing, asking for favors, etc. enables the suitable expression of feelings, desires or opinions, promotes successful management of criticism, and minimizes the 
probability of interpersonal conflict, allowing people to relate to others in an effective and mutually satisfactory way. As social skills are learned and have radical consequences on the social-emotional health of children and adolescents, psychological research has devoted considerable effort to study what factors may facilitate their development and consolidation, as well as other dimensions of social competence such as assertiveness and prosociality. Reviewing the available literature, it can be seen that empathy has been one of the most largely studied personal precursor in relation to child and adolescent social functioning. Empirical research has shown that this variable acts as a strong motivator of prosocial behavior, social responsibility, cooperative conflict resolution, altruistic behavior and general help; while it negatively correlates with social inadequacy, antisocial behavior, aggression and isolation of children and adolescents. Probably for this reason, it is argued that empathy can also be the basis for the establishment of the social skills, and a requirement to properly master these interaction skills. However, although there exists ample evidence of the relationship between empathy and some negative aspects of social skills, such as aggression, there are very few studies that have specifically explored the dynamics between empathy and appropriate social skills. On the other hand, although the predominance of empathy on social functioning is undeniable and its study has been favored by many researchers, some evidence suggets that positive emotions may have a similar powerful influence on the development of certain skills and social skills. Specifically, in the stages of childhood and adolescence, it has been found that joy and sympathy prevent peer rejection; that sympathy and serenity ease assertive and prosocial responses, while decreasing aggressive behaviors; that gratitude is also negatively related to aggression, and that together with personal satisfaction and serenity, it predicts a successful management of interpersonal conflicts. However, while the motivational role of empathy has been widely discussed, the influence of positive emotions on social skills, and specifically on the social skills has not yet reached the attention it deserves. The aim of this study was to include both variables in a model of analysis of variance to investigate the statistical weight that each of them has, and their possible interaction on the appropriate social skills in childhood. A empirical comparative and cross study was developed. This work included 406 Argentine children (227 females and 179 males), mainly between the ages of 10 and 12 years old $(M=11.11 ; D E=1.04)$, who completed the Appropriate Social Skills Subscale of The Matson Evaluation of Social Skills of Youngsters (MES SY) of Matson, Rotatori and Helsel (1983), adapted in Argentinean population by Schulz (2008); The Multidimensional Scale of Empathy for Argentine children, developed by Richaud de Minzi, Lemos \& Oros (2013); and the Child Questionnaire of positive emotions, created by Oros (2014). To analyze the joint action of positive emotions and empathy on the appropriate social skills, 3 (high, moderate and low positive emotionality) x 3 (high, moderate and low empathy) Analysis of Variance (ANOVA) factorial designs were used. The results indicated that positive emotions and empathy facilitate the performance of socially skilled behaviors, being greater the impact of positive emotions compared to empathy. These results are important in understanding the dynamics of the processes involved, and for the further design of intervention programs to promote social skills in childhood.

Key words: Social skills; Empathy; Positive emotions; Children.

\section{INTRODUCCIÓN}

Las habilidades sociales (HHSS) comprenden un eslabón particular dentro de un constructo multidimensional más amplio denominado competencia social (Monjas, 2000). Estas habilidades son predominantemente conductuales, se adquieren en el transcurso de la vida y se ponen en juego en la interacción con otros (Caballo, 2005; Monjas \& González, 2000). Pueden ser verbales o no verbales, pero siempre implican iniciativas apropiadas y efectivas (Michelson, Sugai, Wood \& Kazdin, 1987), tales como: (a) mantener contacto visual con otra persona durante 
una conversación, (b) dar y recibir cumplidos, (c) ayudar a otros, (d) compartir algo, (e) dar las gracias, (f) pedir permiso, (g) disculparse, (h) solicitar favores, etc. (Goldstein, Sprfkin, Gershaw \& Klein, 1989).

La puesta en marcha de estas habilidades posibilita la expresión adecuada de sentimientos, deseos u opiniones, favorece el manejo acertado de la crítica y minimiza la probabilidad de conflictos interpersonales $(\mathrm{Ca}-$ ballo, 2005; Monjas \& González, 2000), permitiendo que las personas se relacionen con otros de una manera efectiva y mutuamente satisfactoria (Monjas, 2000), dando cuenta de un comportamiento adaptativo (Reyna \& Brussino, 2009).

Este comportamiento adaptativo será considerado como tal en función de las normas sociales y culturales que caracterizan el contexto en el cual se desarrolla la conducta.

"Toda habilidad social debe de considerarse dentro de un marco cultural determinado, y los patrones de comunicación variarán ampliamente entre culturas y dentro de una misma cultura, dependiendo de factores tales como la edad, el sexo, la clase social y la educación. Además, el grado de eficacia mostrado por una persona dependerá de lo que desee lograr en la situación particular en que se encuentre. La conducta considerada apropiada en una situación puede ser, obviamente, inapropiada en otra" (García, 2010, p. 225).

La niñez es un período crítico para el desarrollo de las HHSS, y si bien los niños ya nacen con cierto sesgo temperamental hacia la inhibición o la expresividad, estas predisposiciones innatas interactúan con los aprendizajes cotidianos para dar lugar a las manifestaciones sociales observables (Caballo, 2005). Debido a que las HHSS se aprenden y tienen consecuencias radicales en la salud socioemocional de los niños y adolescentes (Contini, 2008; Ipiña, Molina \& Reyna, 2010; Lacunza, 2009; Lacunza, Castro-Solano \& Contini, 2009), la investigación psicológica ha dedicado un considerable esfuerzo a estudiar qué factores pueden propiciar su desarrollo y con- solidación, así como el de otras dimensiones de la competencia social, como la prosocialidad y la asertividad.

Revisando la literatura disponible se puede apreciar que uno de los precursores personales más extendidamente estudiados con relación al funcionamiento social infantil y adolescente ha sido la empatía (cf., Carlo, Raffaelli, Laible \& Meyer, 1999; Eisenberg \& Fabes, 1991; Eisenberg \& Miller, 1987; Feshbach, 1984; Findlay, Girardi \& Coplan, 2006; McMahon, Wernsman \& Parnes, 2006; Mestre, Frías \& Samper, 2004; Mestre, Samper, Tur, Cortés \& Nácher, 2006). Esta habilidad humana, que entreteje tanto aspectos cognitivos como afectivos, resulta fundamental para el desenvolvimiento social adecuado (Eisenberg, 2002). En su dimensión cognitiva implica la capacidad de poder situarse en el lugar del otro y de comprender su punto de vista; mientras que en su dimensión afectiva, implica captar y compartir el estado emocional de la otra persona. Además, la capacidad empática involucra una delicada distinción consciente entre el estado emocional propio y el de los demás (Eisenberg \& Strayer, 1987).

De acuerdo a Decety y Jackson (2004) la empatía estaría conformada por cuatro componentes específicos: la toma de perspectiva (que consiste en imaginar la experiencia del otro), la correspondencia afectiva (que comprende reacciones automáticas basadas en la observación del estado del otro), la autoconciencia afectiva (que implica la habilidad para identificar la propia experiencia y distinguirla de la experiencia ajena) y la regulación empática (que consiste en regular la intensidad de la experiencia afectiva sin sentirse abrumado por ésta).

La investigación empírica haciendo uso de diferentes modelos teóricos y operacionales, ha demostrado que esta variable actúa como un fuerte motivador del comportamiento prosocial (Mestre, Samper \& Frías, 2002; Mestre et al., 2006), de la responsabilidad social (Sanmartín, Carbonell \& Baños, 2011), de la resolución cooperativa de conflictos (Garaigordobil \& Maganto, 2011) y de las conductas altruistas y de ayuda en gene- 
ral (Garaigordobil \& García de Galdeano, 2006; Ortiz, Apodaka, Etxewberría, Eceiza, Fuentes \& López, 1993; Roche, 1982); al mismo tiempo que correlaciona negativamente con la inadecuación social (Gilar, Miñano \& Castejón, 2008), la conducta antisocial, la agresividad y el aislamiento de niños y adolescentes (Garaigordobil \& García de Galdeano, 2006; Findlay et al., 2006). Probablemente por ello, se afirma que la empatía puede también ser la base para el establecimiento de las HHSS y un requisito para dominar adecuadamente estas capacidades de interacción (Lacunza, 2009). No obstante, y aunque existen sobradas pruebas de la relación entre la empatía y algunos aspectos negativos de las HHSS, como la agresión, son muy escasos los estudios que han explorado específicamente la dinámica entre la empatía y las HHSS adecuadas.

Por otra parte, si bien la preeminencia de la empatía sobre el funcionamiento social es innegable y su estudio ha sido privilegiado por muchos investigadores, algunos antecedentes permiten pensar que las emociones positivas podrían tener un influjo similarmente potente en el desarrollo de ciertas competencias y HHSS.

Estudios previos dan cuenta de que las emociones positivas constituyen un factor protector de la salud porque promueven el desarrollo de importantes recursos cognitivos, comportamentales, sociales y biológicos que posibilitan un ajuste funcional al medio (Fredrickson \& Branigan, 2005; Froh, Yurkewicz \& Kashdan, 2008; Johnson, Waugh \& Fredrickson, 2010; Ren, Hu, Zhang \& Huang, 2010; Wood, Maltby, Gillett, Linley \& Joseph, 2008).

Específicamente en la etapa de la niñez y adolescencia se ha encontrado que la alegría y la simpatía protegen del rechazo de los pares; que la simpatía y la serenidad facilitan las respuestas asertivas y prosociales, mientras disminuyen las conductas agresivas; que la gratitud también se relaciona negativamente con la agresividad, y que junto con la satisfacción personal y la serenidad, predice un manejo exitoso de los conflictos interpersonales (Cuello \& Oros, 2014; de la Vega \&
Oros, 2013; Giqueaux \& Oros, 2008; Lemos, Hendrie \& Oros, 2013; Oros, 2008; Richaud de Minzi \& Oros, 2009).

Sin embargo, mientras que el papel motivacional de la empatía ha sido ampliamente abordado, la influencia de las emociones positivas sobre las competencias sociales, y específicamente sobre las HHSS, no ha alcanzado aún la atención que merece.

El objetivo del estudio realizado fue incluir ambas variables en un modelo de análisis de variancia para indagar el peso estadístico que tiene cada una, y su posible interacción, sobre las HHSS apropiadas en la niñez. Se hipotetiza una influencia significativa y similarmente potente de la empatía y de las emociones positivas sobre las HHSS. Con respecto a la interacción, no hay antecedentes que permitan generar hipótesis fuertes acerca del comportamiento conjunto de estas variables sobre las HHSS infantiles; no obstante se podría inferir que su acción conjunta favorece el desarrollo de la variable dependiente. Se espera que los análisis propuestos permitan esclarecer la contribución de estos procesos, otorgando especial énfasis al papel de las emociones positivas.

\section{Metodología \\ PARTICIPANTES}

El estudio es de carácter empírico, comparativo y transversal. Participaron voluntariamente 406 niños (179 varones y 227 niñas) argentinos que asistían a ocho escuelas de la provincia de Misiones (Argentina). Las edades oscilaron mayoritariamente entre los 10 y los 12 años $(M=11.11 ; D E=1.04)$, observándose un porcentaje menor al $10 \%$ de niños que aún no había cumplido los 10 años o que ya había superado los 12 (ver Tabla 1). Las instituciones educativas fueron seleccionadas por disponibilidad, según fue aprobada su participación por parte de las autoridades correspondientes (directivos y supervisores zonales). Las escuelas incluidas son tanto de gestión privada como pública, se sitúan en zonas urbanas y convocan a alumnos de clase media y media-baja. 
El muestreo de los niños se realizó bajo un procedimiento no aleatorio, intencional, considerando como criterios de inclusión que los mismos: (a) estuvieran cursando regularmente desde quinto hasta séptimo grado de la Educación General Básica, (b) contaran con el consentimiento informado de sus padres o tutores legales para participar del estudio, (c) se encontraran presentes el día de la evaluación y (d) desearan participar libre y voluntariamente de esta instancia.

\section{INSTRUMENTOS}

\section{1.- Subescala de Habilidades Sociales Ade- CUADAS}

Este instrumento forma parte de la validación argentina realizada por Schulz (2008) para la Escala de Habilidades Sociales de Matson, Rotatori y Helsel (1983). Está compuesta por 22 ítemes que se puntúan en una escala de cuatro puntos (Nunca, $A$ veces, $A$ menudo, Siempre). La subescala aporta un valor general de HHSS apropiadas, entre las que se incluyen: mirar a los ojos, decir gracias, compartir, colaborar con otros, etc. La consistencia interna de la prueba evaluada mediante el coeficiente Alpha de Cronbach, resultó satisfactoria tanto en la muestra original de estudio $(\alpha=.87)$ como en la muestra de la investigación realizada $(\alpha=.88)$.

2.- Cuestionario Multidimensional de Empatía PARA NIÑOS DE 9 a 12 AÑOS (RICHAUd DE MINZI, LEMOS \& OROS, 2013)

Se trata de una versión preliminar de autorreporte diseñada para niños de 9 a 12 años de edad y está basada en el modelo de Decety y Jackson (2004). Incluye 17 ítemes escalados en cuatro puntos (Siempre, Muchas veces, Pocas veces, Nunca) para evaluar las siguientes dimensiones: (a) Toma de perspectiva (por ejemplo, Aunque otro piense distinto a mí, puedo entenderlo), (b) Correspondencia afectiva con las emociones o acciones de otros (por ejemplo, Cuando estoy con alguien que está triste yo también me pongo triste), (c) Autoconciencia (por ejemplo, Me doy cuenta cuando estoy triste...) y (d) Autorregulación empática (por ejemplo, Cuando uno de mis amigos está triste yo también me pongo triste pero igual puedo seguir haciendo mis cosas.). La escala muestra un buen funcionamiento factorial, presenta índices entre bajos y medianamente adecuados de consistencia interna (valores de Alpha de Cronbach entre .54 y .65) (Richaud de Minzi et al., 2013). En nuestra muestra, el coeficiente $\mathrm{Al}$ pha de Cronbach para la prueba completa fue aceptable (.77), resultando al igual que en el trabajo original, entre bajo y medianamente adecuado para cada una de las dimensiones (Correspondencia afectiva: .68, Autoconciencia: .63, Toma de perspectiva: .67 y Autorregulación empática: .50). El valor más bajo de consistencia interna corresponde a la Escala de Autorregulación que evalúa en una sola dimensión la capacidad del niño para regular muy diferentes estados afectivos (alegría, tristeza, enojo, tensión, etc.); diversidad que podría explicar la baja homogeneidad de los puntajes. A pesar de que la escala evidencia una buena validez de constructo, se considera aún en fase experimental y está siendo objeto de nuevos estudios y redefiniciones con el fin de poder aumentar sus valores de consistencia interna (Richaud de Minzi et al., 2013).

3.- Cuestionario Infantil de Emociones PositiVAS (CIEP - OROS, 2014)

Este cuestionario comprende 23 ítemes que se responden con una de las siguientes opciones: (3) sí, (2) más o menos, o (1) no, según el grado de acuerdo con la frase que se expresa. El cuestionario puede aplicarse a niños de 8 a 12 años de edad, y proporciona información acerca de cinco emociones positivas: alegría, serenidad, gratitud, satisfacción personal y simpatía. En el estudio original el Alpha de Cronbach para la prueba completa fue igual a .95 y los coeficientes para cada emoción oscilaron entre .66 y .93 (Oros, 
2014). En la muestra estudiada, la consistencia interna para todos los ítemes fue muy buena (.80), mientras que los valores correspondientes a las dimensiones resultaron más bien bajos (entre .55 y .68).

Procedimientos de RECOLECCIÓN Y ANÁLISIS DE DATOS

Se solicitó la autorización de los directivos y docentes de las diferentes escuelas para administrar las pruebas de evaluación en el horario habitual de clases. La actividad se realizó de manera colectiva, dentro del aula, en una única oportunidad. La participación de los niños fue voluntaria y se contó con el consentimiento informado de sus padres o tutores legales. La nota de consentimiento informado, que fue enviada mediante el cuaderno oficial de comunicaciones, incluía una breve descripción del estudio y garantizaba la participación voluntaria, el trato respetuoso y confidencial de las respuestas y la utilización de los datos con fines exclusivos de investigación.

Para analizar la acción conjunta de las emociones positivas y la empatía sobre las HHSS apropiadas, se ejecutó un ANOVA factorial con un diseño 3 (alta, moderada y baja emocionalidad positiva) x 3 (alta, moderada y baja empatía). La variable dependiente estuvo constituida por los valores brutos de la subescala de HHSS apropiadas. Las variables independientes fueron categorizadas en tres niveles (puntajes altos, moderados y bajos) mediante el cálculo de percentiles, estableciendo dos puntos de corte: 33.33 y 66.66 puntos. Las comparaciones de medias post hoc se realizaron con el método de Scheffé.

\section{Resultados}

A continuación se presentan los resultados obtenidos. Se exponen en primer lugar los efectos de los puntajes brutos de la empatía y de las emociones positivas sobre las HHSS. En segundo lugar se describen los efectos de cada dimensión de las variables independientes y sus correspondientes contrastes post hoc. En tercer lugar se presentan los resultados en función del género y la edad de los niños.

Se encontraron efectos principales significativos de la empatía $[F(2,390)=10.28$; $\left.p<.001 ; \eta^{2} p=.05\right]$ y de las emociones positivas $\left[F(2,390)=20.75 ; p<.001 ; \eta^{2} p=.10\right]$, siendo mayor la importancia práctica de este efecto para las emociones positivas, como lo muestran los valores de eta cuadrado parcial. La interacción entre ambas variables no resultó significativa $[F(4,390)=.94 ; p=.440$; $\left.\eta^{2} p=.01\right]$.

Para estudiar cuáles eran los aspectos de la empatía y cuales las emociones positivas que en particular incidían sobre el comportamiento social de los niños se realizaron ANOVAS factoriales incluyendo por separado las dimensiones de cada variable independiente. Debido a que los valores de consistencia interna de estas dimensiones resultaron más bien bajos en este estudio, los siguientes análisis se ejecutaron a modo exploratorio y por lo tanto los resultados deben considerarse como una primera aproximación al tema, siendo necesaria su comprobación en otras muestras de niños.

Con respecto a las dimensiones de la empatía, se encontró que la respuesta afectiva $\left[F(2,320)=5.68 ; p=.004 ; \eta^{2} p=.03\right]$, la autoconciencia emocional $[F(2,320)=10.14$; $\left.p<.001 ; \eta^{2} p=.06\right]$ y la regulación empática $\left[F(2,326)=5.59 ; p=.004 ; \eta^{2} p=.03\right]$ inciden significativamente sobre las HHSS, siendo la autoconciencia emocional la única dimensión con tamaño de efecto considerable. La toma de perspectiva no resultó significativa $[F(2,320)=2.59 ; p=.077$; $\left.\eta^{2} p=.02\right]$. Tampoco se observaron interacciones significativas entre las dimensiones.

Según los contrastes post hoc, las diferencias más importantes se aprecian entre los grupos extremos (alta y baja empatía). A mayor empatía, en cualquiera de las dimensiones, corresponde mayor despliegue de HHSS apropiadas (ver Tabla 2).

Con respecto a las emociones positivas, se encontró que la alegría $[F(2,308)=6.23$; $\left.p=.002 ; \eta^{2} p=.04\right]$, la gratitud $[F(1$, 
$\left.308)=16.10 ; p<.001 ; \eta^{2} p=.05\right]$ y la simpatía $\left[F(2,308)=6.14 ; p=.002 ; \eta^{2} p=.04\right]$ jugarían el rol más importante entre las cinco emociones evaluadas, aunque con tamaños de efecto entre moderados y moderadamente bajos. La influencia de la serenidad $[F(2$, $\left.308)=2.70 ; p=.070 ; \eta^{2} p=.02\right]$ y de la satisfacción personal $[F(1,308)=.07$; $\left.p=.786 ; \eta^{2} p=.00\right]$ no resultó significativa. Unas pocas interacciones alcanzaron valores de significación menores a .05 pero con tamaños de efecto bajos (entre .02 y .03) por lo que fueron consideradas espurias.

De acuerdo a los contrastes post hoc, las diferencias más marcadas en las HHSS infantiles se producen entre los grupos extremos de las emociones positivas. A medida que aumenta la emocionalidad positiva de los niños mejoran sus valores de HHSS (ver Tabla 3).

Estos mismos análisis se ejecutaron para las submuestras de niñas y varones por separado, encontrando que en general los resultados se mantienen estables. Las emociones positivas y la empatía favorecen las HHSS independientemente del género de los niños. Se observan sin embargo algunas diferencias sutiles que parecen indicar que en el grupo de las niñas, las emociones positivas jugarían un rol más relevante que la empatía en la ejecución de las HHSS adecuadas, mientras que para los varones, cobraría algo más de importancia la empatía, aunque la diferencia en este caso ha sido mucho más tenue (ver Tablas 4 y 5 ).

Por otra parte, también se replicaron los análisis pero extremando previamente los grupos según la edad: niños y niñas de cuarto grado por un lado $(M$ de edad $=9.95 ; D E=.22)$, y niños y niñas de séptimo grado, por otro ( $M$ de edad $=12.28 ; D E=.59)$. Se comprobó que los resultados se mantienen similarmente estables independientemente de la edad. Pero nuevamente se observan algunas ligeras diferencias entre los grupos. Mientras que en los niños menores es más potente la influencia de las emociones positivas al momento de ejecutar sus HHSS, en los mayores, cobra más importancia la empatía (ver Tablas 6 y 7$)$.

\section{DISCUSIÓN Y CONCLUSIONES}

El propósito de este estudio ha sido arrojar luz sobre una cuestión poco examinada. Porque si bien abundan los estudios que analizan y afirman el rol preponderante de la empatía sobre los comportamientos sociales, la influencia de las emociones positivas ha sido menos tenida en cuenta a pesar de sus meritorias implicaciones en la salud psicosocial de los niños.

Los resultados obtenidos confirman la hipótesis de que tanto la empatía como las emociones positivas son importantes precursores de las HHSS adecuadas en la niñez. Los niños empáticos, especialmente aquellos que manifiestan respuestas afectivas apropiadas, buena autoconciencia emocional y alta regulación empática, tienen una probabilidad mayor de poner en marcha HHSS adecuadas. Estos resultados están en línea con los antecedentes revisados (Lacunza, 2009).

En relación a la toma de perspectiva, los resultados no fueron significativos, por lo tanto se concluye que el proceso cognitivo de poder situarse en el lugar del otro, no representa en esta muestra de niños, un elemento relevante a la hora de explicar las HHSS. En estudios anteriores sí se han observado correlaciones de este aspecto de la empatía con otras conductas de índole social, como el comportamiento prosocial en adolescentes (Mestre et al., 2002), aunque, coincidentemente, las correlaciones con los aspectos emocionales resultaron más fuertes.

Por otra parte, con respecto al papel de las emociones positivas, se observó que los niños alegres, simpáticos y agradecidos tienen mayor facilidad para desplegar conductas sociales adecuadas en sus interacciones con pares y adultos. Es interesante destacar que las tres emociones que han resultado influyentes de las habilidades sociales (simpatía, gratitud y alegría) tienen una fuerte impronta social, a diferencia de la satisfacción personal o la experiencia de serenidad, que son emociones más centradas en uno mismo.

La simpatía es la emoción positiva que acompaña los procesos cognitivos de la empatía. Aunque en ocasiones se han utilizado 
como intercambiables, los conceptos de simpatía y empatía no son equivalentes. Desde un punto de vista estricto, la empatía no es una emoción positiva sino una habilidad general que engloba a la simpatía y a los procesos cognitivos de reconocimiento emocional y toma de perspectiva, entre otros. Puntualmente, la simpatía implica la sensibilidad para con las emociones y necesidades de otras personas, comprende el fenómeno de la sintonía emocional y el deseo de aliviar el dolor ajeno (Oros, 2011). Esta condición emocional elevaría la disposición a desplegar conductas sociales de ayuda y asistencia hacia quienes se encuentran en dificultad, o de compartir lo que se tiene con los demás, exteriorizando de este modo un comportamiento social positivo. Por su parte, la gratitud es una emoción que surge cuando el niño reconoce que alguien le ha concedido intencionalmente un favor que ha contribuido a su bienestar personal (Chesney et al., 2005; Emmons, Mc Cullough \& Tsang, 2003; Lazarus, 2000; Strümpfer, 2006). Es esperable por lo tanto que aquellos niños que han desarrollado una genuina experiencia de gratitud se vean movilizados a desplegar conductas sociales adecuadas, especialmente de agradecimiento y retribución, cuando alguien los beneficia (Emmons \& Shelton, 2002). Finalmente, la alegría es un estado emocional que se caracteriza por una intensa sensación de agrado y regocijo (Herring, Burleson, Roberts \& Devine, 2011; Lazarus, 2000) y por impulsar a los niños a jugar (Fredrickson \& Joiner, 2002). La alegría intensificaría la confianza y prepararía al niño para que experimente y fomente vínculos sociales mediante el contagio de sensaciones positivas (Richaud de Minzi et al., 2005). Además, la risa que surge como parte inherente de este estado afectivo, es un indicador facial muy importante que funciona como señal de apertura hacia nuevas interacciones amistosas (Fredrickson \& Cohn, 2008). Algunos de los comportamientos sociales evaluados en este estudio implican precisamente la capacidad de divertir o hacer reír a los demás, de expresar abiertamente los sentimientos y de reírse de los chistes e historias divertidas que cuentan otros, aspectos que naturalmente serán más fáciles de manifestar para aquellos niños predominantemente alegres.

Con respecto a la interacción entre las variables, se puede decir que el resultado de la combinación de la empatía y las emociones positivas no difiere de la suma de los efectos principales de ambas variables. Por lo que al parecer estos atributos psicológicos, si bien relacionados entre sí, actuarían de manera independiente sobre las HHSS. Estos resultados refutan la conjetura inicialmente propuesta. Debido a que resultan insuficientes los antecedentes teóricos que permitan ampliar estos resultados, se sugiere profundizar el tema en futuras investigaciones.

Resultó novedoso que el impacto de las emociones positivas sobre las HHSS superara al de la empatía. Parecería que son los aspectos emocionales de la organización psíquica los que inciden con mayor efecto sobre las HHSS, constituyendo en sí mismos una base fecunda para su desarrollo. Esta hipótesis, que deberá ser analizada con mayor detenimiento en próximos estudios, recibe cierto apoyo de investigaciones previas en las que se analizó la influencia de la empatía sobre las capacidades sociales de adolescentes encontrando que son precisamente los aspectos afectivos de la misma los que aportan el mayor peso explicativo. En otras palabras, y coincidiendo con Sroufe, Schork, Motti, Lawroski y LaFreniere (1984), cabe pensar que la emoción tendría un rol dominante en la organización de las conductas de interacción social, por encima de otros procesos psicológicos implicados.

De todas maneras, cabe resaltar que esta predominancia afectiva podría estar moderada por el género y la edad. Mientras que para el despliegue de HHSS de las niñas cobrarían más relevancia las emociones positivas, para los varones adquiriría más importancia la empatía. ¿A qué pueden deberse estos resultados? Probablemente a que las niñas, por una cuestión de normativa social asociada al rol sexual, sean predominantemente emotivas, es decir, tengan una prevalencia hacia los aspectos afectivos por sobre los racionales (Retuerto, 2004) al momento 
de ejecutar ciertos comportamientos específicos en una interacción social. Y precisamente, de las dos variables independientes incluidas en este estudio, la empatía es la que tiene mayor componente cognitivo y las emociones positivas mayor representación de aspectos netamente afectivos. Es de esperar entonces, que para las niñas sean los sentimientos de compasión, ternura, gratitud y alegría los que expliquen más fuertemente sus habilidades de interacción social con otros, a diferencia de los varones que tenderían a emplear más las capacidades cognitivas de autoconciencia y algo de toma de perspectiva como lo dejó ver un estudio previo (Oros \& Fontana, 2013).

Por otro lado, mientras que para los niños menores son más influyentes las emociones positivas al momento de ejecutar sus HHSS, para los mayores cobra una importancia más acentuada la empatía, especialmente la dimensión de regulación emocional, que precisamente está más consolidada a las puertas de la adolescencia. La autorregulación se refiere a la habilidad de controlar y modular la intensidad de las expresiones emocionales (positivas o negativas) y de interactuar con otros de maneras cada vez más complejas de acuerdo a reglas sociales. Es esperable entonces que este proceso cobre más relevancia a medida que aumenta la edad.

A pesar de que estos resultados tienen respaldo teórico, es necesario continuar indagando este tema antes de extraer conclusiones definitivas. ¿Será que las emociones positivas evaluadas aquí han mostrado un rol predominante porque por definición están muy asociadas a los procesos empáticos? ¿o será que la empatía ha mostrado siempre un rol preponderante porque involucra indefectiblemente la experiencia de ciertas emociones positivas? Estos interrogantes sugieren la necesidad de seguir explorando la temática, en diferentes muestras y con diferentes instrumentos de evaluación para arrojar luz a cuestiones aún no suficientemente analizadas. De todas maneras, lo hasta aquí presentado aporta conocimiento nuevo, sugiriendo que la empatía y las emociones positivas podrían funcionar como excelentes vehículos para la promoción de las HHSS en la niñez, por lo que sería bueno tener en cuenta su injerencia al momento de diseñar programas de intervención en HHSS.

Entre las limitaciones del trabajo realizado, se cuentan principalmente los valores bajos de consistencia interna observados en las subescalas utilizadas, lo cual no permite extraer conclusiones definitivas con respecto al papel de los distintos aspectos de la empatía y de las cinco emociones positivas, sobre las HHSS de los niños. Por otra parte, es importante resaltar que en este estudio sólo se consideró un puntaje total de HHSS, resultando un desafío para próximas investigciones obtener una medición de diferentes aspectos de este constructo para poder analizar de qué manera se ve afectado cada uno de ellos por las emociones positivas y la empatía. 
TABLA 1

DISTRIBUCIÓN DE LA MUESTRA SEGÚN GÉNERO Y EDAD

\begin{tabular}{|c|c|c|c|c|}
\hline Género & 10 años & $\begin{array}{c}\text { Edad }^{1} \\
11 \text { años }\end{array}$ & 12 años & Total \\
\hline Niñas & 70 & 72 & 85 & 227 \\
\hline Varones & 65 & 54 & 60 & 179 \\
\hline Total & 135 & 126 & 145 & 406 \\
\hline
\end{tabular}

${ }^{1}$ La minoría de niños que estaba próxima a cumplir los 10 años o que había superado los 12, fue incluida en las categorías de edad más cercana (10 y 12 años, respectivamente).

TABLA 2

MEDIAS Y CONTRASTES POST HOC PARA LAS HABILIDADES SOCIALES EN FUNCIÓN DE LAS DIMENSIONES DE EMPATÍA

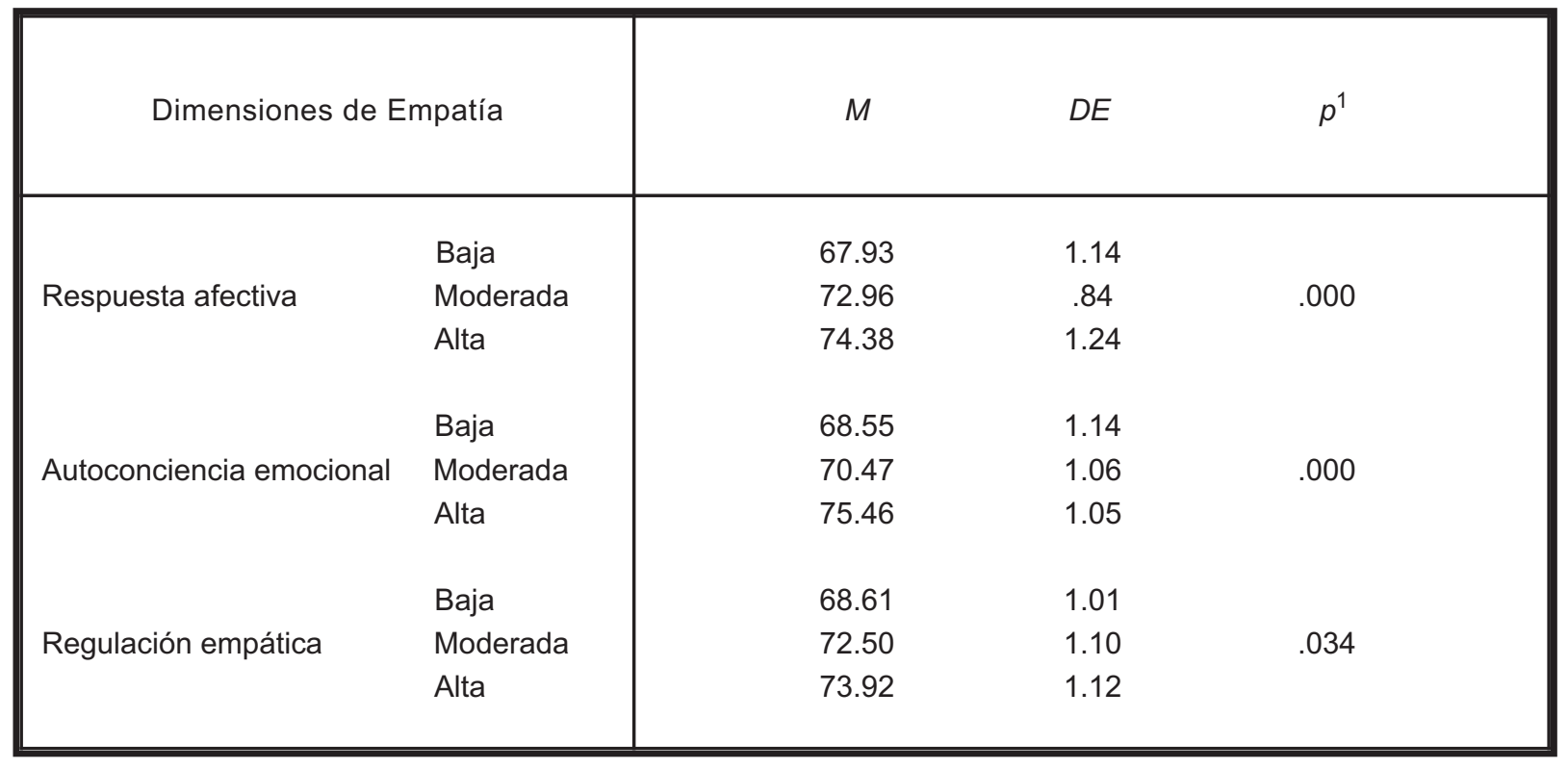

${ }^{1}$ Los valores de significación corresponden a las comparaciones de los grupos extremos (alta y baja empatía). 
TABLA 3

MEDIAS Y CONTRASTES POST HOC PARA LAS HHSS EN FUNCIÓN DE LAS EMOCIONES POSITIVAS

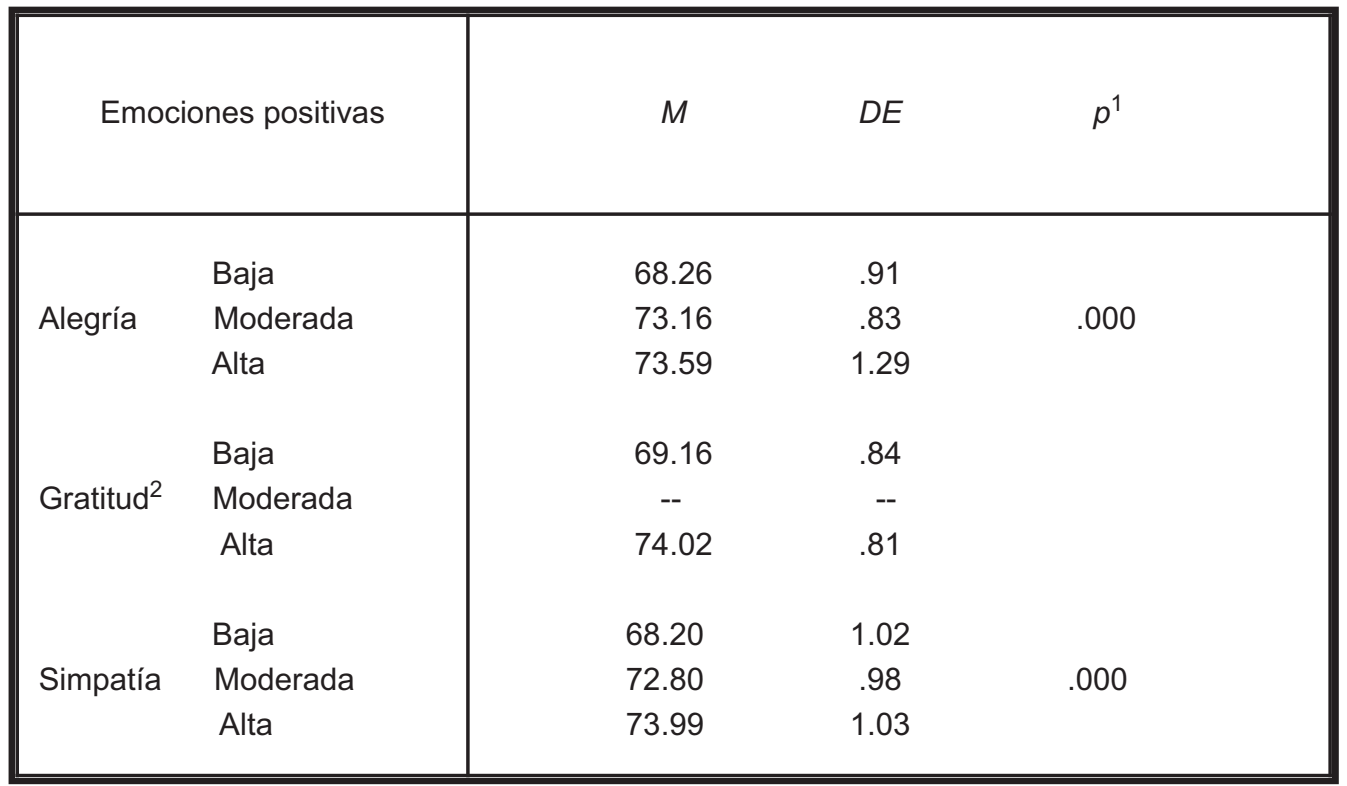

${ }^{1}$ Los valores de significación corresponden a las comparaciones de los grupos extremos (alta y baja emocionalidad positiva).

${ }^{2}$ No se registraron valores moderados de Gratitud.

TABLA 4

RESULTADOS DEL ANÁLISIS DE VARIANCIA CORRESPONDIENTE A LAS SUBMUESTRAS SEGÚN EL GÉNERO

\begin{tabular}{|c|c|c|c|c|c|}
\hline & & $F$ & $p$ & $\eta^{2} p$ & $n$ \\
\hline \multirow{3}{*}{ Niñas } & Empatía & 3.68 & .027 & .03 & \multirow{3}{*}{227} \\
\hline & Emociones positivas & 15.15 & .000 & .12 & \\
\hline & Empatía x Emoc. positivas & .40 & .811 & .01 & \\
\hline \multirow{3}{*}{ Varones } & Empatía & 7.20 & .001 & .08 & \multirow{3}{*}{$17 \varsigma$} \\
\hline & Emociones positivas & 5.01 & .008 & .06 & \\
\hline & Empatía x Emoc. positivas & 1.10 & .356 & .02 & \\
\hline
\end{tabular}


TABLA 5

MEDIAS Y CONTRASTES POST HOC DE LAS HHSS PARA NIÑAS Y VARONES, EN FUNCIÓN DE SU EMPATÍA Y EMOCIONES POSITIVAS

\begin{tabular}{|c|c|c|c|c|}
\hline & & $M$ & $D E$ & $p^{1}$ \\
\hline $\begin{array}{ll} & \text { Empatía } \\
\text { Niñas } & \\
& \text { Emoción positiva }\end{array}$ & $\begin{array}{l}\text { Baja } \\
\text { Moderada } \\
\text { Alta } \\
\text { Baja } \\
\text { Moderada } \\
\text { Alta }\end{array}$ & $\begin{array}{l}68.51 \\
71.64 \\
73.08 \\
\\
66.02 \\
71.85 \\
75.37\end{array}$ & $\begin{array}{c}1.21 \\
.97 \\
1.26 \\
\\
\\
1.25 \\
1.03 \\
1.17\end{array}$ & $\begin{array}{l}.000 \\
.000\end{array}$ \\
\hline $\begin{array}{l}\text { Empatía } \\
\text { Varones } \\
\text { Emoción positiva }\end{array}$ & $\begin{array}{l}\text { Baja } \\
\text { Moderada } \\
\text { Alta } \\
\text { Baja } \\
\text { Moderada } \\
\text { Alta }\end{array}$ & $\begin{array}{l}66.23 \\
71.50 \\
74.80 \\
\\
66.79 \\
70.98 \\
74.77\end{array}$ & $\begin{array}{l}1.30 \\
1.30 \\
2.20 \\
\\
\\
2.07 \\
1.29 \\
1.50\end{array}$ & .000 \\
\hline
\end{tabular}

${ }^{1}$ Por razones de espacio, sólo se registra el valor de significación asociado a los contrastes post hoc entre los grupos extremos, sin embargo todas las comparaciones realizadas fueron significativas $(p<.05)$.

TABLA 6

Resultados del ANÁlisis de VARIANCIA CORRESPONDIENTE A LAS SUBMUESTRAS SEGÚN LA EDAD

\begin{tabular}{|c|c|c|c|c|c|}
\hline & & $F$ & $p$ & $\eta^{2} p$ & $n$ \\
\hline \multirow{3}{*}{ Menores } & Empatía & 2.72 & .070 & .04 & \multirow{3}{*}{135} \\
\hline & Emociones positivas & 18.06 & .000 & .22 & \\
\hline & Empatía x Emociones positivas & 1.72 & .150 & .05 & \\
\hline \multirow{3}{*}{ Mayores } & Empatía & 9.12 & .000 & .12 & \multirow{3}{*}{145} \\
\hline & Emociones positivas & 4.99 & .008 & .07 & \\
\hline & Empatía $\times$ Emociones positivas & 1.59 & .181 & .05 & \\
\hline
\end{tabular}


TABLA 7

MEDIAS Y CONTRASTES POST HOC DE LAS HHSS PARA LAS SUBMUESTRAS SEGÚN EDAD, EN FUNCIÓN DE SU EMPATÍA Y EMOCIONES POSITIVAS

\begin{tabular}{|c|c|c|c|c|}
\hline & & $M$ & $D E$ & $p^{1}$ \\
\hline \multicolumn{5}{|l|}{ Menores } \\
\hline & Baja & 69.35 & 1.16 & \\
\hline \multirow{3}{*}{ Empatía } & Moderada & 72.32 & 1.17 & .000 \\
\hline & Alta & 73.83 & 1.85 & \\
\hline & Baja & 65.12 & 1.67 & \\
\hline \multirow[t]{2}{*}{ Emoción positiva } & Moderada & 72.83 & 1.37 & .000 \\
\hline & Alta & 77.54 & 1.22 & \\
\hline \multicolumn{5}{|l|}{ Mayores } \\
\hline & Baja & 65.76 & 1.48 & \\
\hline \multirow{2}{*}{ Empatía } & Moderada & 70.25 & 1.23 & .000 \\
\hline & Alta & 74.81 & 1.51 & \\
\hline \multirow{3}{*}{ Emoción positiva } & Baja & 66.75 & 1.43 & \\
\hline & Moderada & 70.84 & 1.27 & .000 \\
\hline & Alta & 73.22 & 1.53 & \\
\hline
\end{tabular}

${ }^{1}$ Por razones de espacio, sólo se registra el valor de significación asociado a los contrastes post hoc entre los grupos extremos, sin embargo todas las comparaciones realizadas fueron significativas $(p<.05)$.

\section{REFERENCIAS BIBLIOGRÁFICAS}

Caballo, V. (2005). Manual de evaluación y entrenamiento de las habilidades sociales [Asssessment and training manual of social skills] (3a. ed.). Madrid: Siglo XXI.

Carlo, G., Raffaelli, M., Laible, D.J. \& Meyer, K.A. (1999). Why are girls less physically aggressive than boys? Personality and parenting mediators of physical aggression. Sex Roles, 40(9/10), 711-729.
Chesney, M., Darbes, L., Hoerster, K., Taylor, J., Chambers, D. \& Anderson, D. (2005). Positive emotions: Exploring the other hemisphere in behavioral medicine. International Journal of Behavioral Medicine, 12(2), 50-58. http://dx. doi.org/10.1207/s15327558ijbm12 02_2

Contini, N. (2008). Las habilidades sociales en la adolescencia temprana: Perspectivas desde la Psicología Positiva [Cognitive abilities in earling adolescence: An outlook from Positive Psychology]. Psicodebate, 9, 15-27. 
Cuello, M. \& Oros, L.B. (2014). Serenity and its relationship to prosocial and aggresive behaviors in Argentinean Children. Journal of Latino-Latin American Studies, 6(1), 31-39.

de la Vega, N. \& Oros, L.B. (2013). El rol de las emociones positivas empáticas en el comportamiento social de los adolescentes [The role of empathic positive emotions in the social behavior of teenagers]. Psicodebate, 13(1), 418.

Decety, J. \& Jackson, P.H. (2004). The functional architecture of human empathy. Behavioural and Cognitive Neuroscience Review, 3(2), 71100. http://dx.doi.org/10.1177/15345823042 67187

Downs, A. \& Smith, T. (2004). Emotional understanding, cooperation, and social behavior in high-functioning children with autism. Journal of Autism and Developmental Disorders, 34(6), 625-635. http://dx.doi.org/10.1007/s10803-00 4-5284-0

Eisenberg, N. (2002). Empathy-related emotional responses, altruism, and their socialization. En R.J. Davidson \& A. Harrington (Eds.), Visions of compassion: Western scientists and Tibetan Buddhists examine human nature (pp. 131164). London: Oxford University Press.

Eisenberg, N. \& Miller, P.A. (1987). The relation of empathy to prosocial and related behaviors. Psychological Bulletin, 101(1), 91-119. http:// dx.doi.org/10.1037/0033-2909.101.1.91

Eisenberg, N. \& Fabes, R.A. (1991). Prosocial behavior and empathy: A multimethod, developmental perspective. En E. Clark (Ed.), Review of Personality and Social Psychology, Vol. 12 (pp. 34-61). Newbury Park: Sage.

Eisenberg, N. \& Strayer, J. (1987). Critical issues in the study of empathy. En N. Eisenberg \& J. Strayer (Eds.), Empathy and its development (pp. 3-15). NY: Cambridge University Press.

Emmons, R.A. \& Shelton, C.M. (2002). Gratitude and the science of Positive Psychology. En C.R. Snyder \& S.J. Lopez (Eds.), Handbook of positive psychology (pp. 459-471). Oxford, England: Oxford University Press.

Emmons, R.A., McCullough, M.E. \& Tsang, J.A. (2003). The assesssment of gratitude. En S. Lopez \& C.R. Snyder (Eds.), A handbook of models and measures. Positive psychological assessment (pp. 327-341). Washington, DC: APA.

Feshbach, N. (1984). Empathy, empathy training and the regulation of aggression in elementary school children. Aggression in Children and Youth, 17, 192-208. http://dx.doi.org/10.1007/ 978-94-017-0665-0_9

Findlay, L.C., Girardi, A. \& Coplan, R.J. (2006). Links between empathy, social behavior, and social understanding in early childhood. Early Childhood Research Quarterly, 21(3), 347359. http://dx.doi.org/10.1016/j.ecresq.2006. 07.009

Fredrickson, B.L. \& Branigan, C. (2005). Positive emotions broaden the scope of attention and thought-action repertoires. Cognition and Emotion, 19(3), 313-332.

Fredrickson, B.L. \& Cohn, M.A. (2008). Positive emotions. En M. Lewis, J.M. Haviland-Jones, \& L.F. Barrett (Eds.), Handbook of emotions (pp.777-796). New York: Guilford Press.

Fredrickson, B.L. \& Joiner, T. (2002). Positive emotions trigger upward spirals toward emotional well-being. Psychological Science, 13 (2), 172-175. http://dx.doi.org/10.1111/1467-9 280.00431

Froh, J.J., Yurkewicz, C. \& Kashdan, T.B. (2008). Gratitude and subjective well-being in early adolescence: Examining gender differences. Journal of Adolescence, 20, 1-18. http://dx. doi.org/10.1016/j.adolescence.2008.06.006

Garaigordobil, M. \& Maganto, C. (2011). Empatía y resolución de conflictos durante la infancia y la adolescencia [Empathy and conflict resolution during childhood and adolescence]. Revista Latinoamericana de Psicología, 43(2), 51-62. 
Garaigordobil, M. \& García de Galdeano, P. (2006). Empatía en niños de 10 a 12 años [Empathy in children aged 10 to 12 years]. Psicothema, 18(2), 180-186.

García, A.D. (2010). Estudio sobre la asertividad y las habilidades sociales en el alumnado de educación social [A study on assertiveness and social skills in students of social education]. Revista de Educación, 12, 225-240.

Gilar, R., Miñano, P. \& Castejón, J.L. (2008). Inteligencia emocional y empatía: Su influencia en la competencia social en educación secundaria obligatoria [Emotional intelligence and empathy: Their influence on the social competence in secondary obligatory education]. Suma Psicológica, 5(1), 21-32.

Giqueaux, G. \& Oros, L. (2008). Las emociones positivas como facilitadoras de la sociabilidad infantil [Positive emotions as facilitators of children's sociability]. Trabajo presentado en el XX Congreso Nacional de Psicodiagnóstico. La Plata, Argentina.

Goldstein, A.P., Sprfkin, R.P., Gershaw, N.J. \& Klein, P. (1989). Habilidades sociales y autocontrol en la adolescencia. Un programa de enseñanza [Social skills and self-control in adolescence. A teaching program]. Barcelona: Martínez Roca.

Herring, D.R., Burleson, M.H., Roberts, N.A. \& Devine, M.J. (2011). Coherent with laughter: subjective experience, behavior, and physiological responses during amusement and joy. International Journal of Psychophysiology, 79, 211-218. http://dx.doi.org/10.1016/j.ijps ycho. 2010.10.007

Ipiña, M.J., Molina, L. \& Reyna, C. (2011). Propiedades psicométricas de la Escala MESSY (versión autoinforme) en niños argentinos [Psychometric Properties of the MESSY Scale (Self-Assessment) in Argentinean children]. Revista de Psicología, 29(2), 245-264.

Izard, C., Fine, S., Schultz, D., Mostow, A., Ackerman, B. \& Youngstrom, E. (2001). Emo- tion knowledge as a predictor of social behavior and academic competence in children at risk. Psychological Science, 12(1), 18-23. http: //dx.doi.org/10.1111/1467-9280.00304

Johnson, K.J., Waugh, C.E. \& Fredrickson, B.L. (2010). Smile to see the forest: Facially expressed positive emotions broaden cognition. Cognition and Emotion, 24(2), 299-321. http:// dx.doi.org/10.1080/02699930903384667

Lacunza, A. (2009). Las habilidades sociales para recursos en el desarrollo de fortalezas en la infancia [Social skills as resources to develop strengths in childhood]. Psicodebate, Psicología, Cultura y Sociedad, 10, 231-248.

Lacunza, A.B., Castro-Solano, A.C., \& Contini, N. (2009). Habilidades sociales preescolares: Una escala para niños de contextos de pobreza [Preschool social abilities: Construction and validation of a scale for children in contexts of poverty]. Psicología, 27(1), 3-28.

Lazarus, R.S. (2000). Estrés y emoción. Manejo e implicaciones en nuestra salud [Stress and emotion. Management and implications on our health]. España: Desclée de Brouwer.

Lemos, V., Hendrie, K. \& Oros, L. (2013). Simpatía y su incidencia en la conducta prosocial en niños de 6 y 7 años [Sympathy and its impact on prosocial behavior in children 6 and 7 years]. Manuscrito presentado para su publicación.

López, F., Apodaca, P., Etxebarria, I., Fuentes, M.J. \& Ortiz, M.J. (1998). Conducta prosocial en preescolares [Prosocial behaviour in preschool children]. Infancia y Aprendizaje, 21(2), 45-61. Matson, J.L., Rotatori, A.F. \& Helsel, W.J. (1983). Development of a Rating Scale to Measure Social Skills in Children: The Matson Evaluation of Social Skills with Youngsters (MES SY). Behaviour Research and Therapy, 21, 335-340. http://dx.doi.org/10.1016/0005-7967 (83)90001-3

McMahon, S.D., Wernsman, J. \& Parnes, A.L. (2006). Understanding prosocial behavior: The 
impact of empathy and gender among African American adolescents. Journal of Adolescent Health, 39(1), 135-137. http://dx.doi.org/10. 1016/j.jadohealth.2005.10.008

Mestre, V., Frías, M.D. \& Samper, P. (2004). La medida de la empatía: Análisis del Interpersonal Reactivity Index [Measuring Empathy: The Interpersonal Reactivity Index]. Psicothe$m a, 16(2), 255-260$.

Mestre, V., Samper, P. \& Frías, M.D. (2002). Procesos cognitivos y emocionales predictores de la conducta prosocial y agresiva: La empatía como factor modulador [Cognitive and emotional processes as predictors of prosocial behaviour and aggressive conduct: Empathy as a modulating factor]. Psicothema, 14, 227-232.

Mestre, V., Samper, P., Tur, A.M., Cortés, M.T. \& Nácher, M.J. (2006). Conducta prosocial y procesos psicológicos implicados: Un estudio longitudinal en la adolescencia [Psychological processes implied by prosocial behavior: A longitudinal study of adolescents]. Revista Mexicana de Psicología, 23(2), 203-215.

Michelson, L., Sugai, D., Wood, R. \& Kazdin, A. (1987). Las habilidades sociales en la infancia [Social skills in childhood]. Barcelona: Martínez Roca.

Monjas, M.I. \& González, B. (2000). Las habilidades sociales en el curriculo [Social skills in the curriculum]. Madrid: Ministerio de Educación.

Monjas, M. I. (2000). Programa de Enseñanza de Habilidades de Interacción Social para Niños y Niñas en Edad Escolar [Teaching Social Interaction Skills Program to Children of School Age]. Madrid: CEPE.

Oros, L.B. (2008). Promoviendo la serenidad infantil en el contexto escolar. Experiencias preliminares en una zona de riesgo ambiental [Promoting calmness in school context. Preliminary experiences in an environmentally atrisk setting]. Interdisciplinaria, 25(2), 181-196.
Oros, L.B. (2011). Nuevas contribuciones para el desarrollo de emociones positivas en niños En M.C. Richaud de Minzi \& V. Lemos (Eds.), Psicología y otras ciencias del comportamiento. Compendio de investigaciones actuales [Psychology and other behavioral sciences. Current research compendium]. Paraná, Entre Ríos: Universidad Adventista del Plata.

Oros, L.B. (2014). Nuevo Cuestionario de Emociones Positivas para Niños [New Questionnaire of Positive Emotions for Children]. Anales de Psicología, 30(2), 522-529.

Oros, L.B. \& Fontana, A. (2013). Los beneficios sociales de la empatía y de las emociones positivas durante la niñez [The social benefits of empathy and positive emotions during childhood]. Trabajo presentado en el XXXIV Congreso Interamericano de Psicología. Brasilia, Brasil.

Ortiz, M.J., Apodaka, P., Etxewberría, I., Eceiza, A., Fuentes, M.J. \& López, F. (1993). Algunos predictores de la conducta prosocial-altruista en la infancia: Empatía, toma de perspectiva, apego, modelos parentales, disciplina familiar e imagen del ser humano [Some predictors of prosocial-altruistic behavior in childhood: Empathy, perspective taking, attachment, parental models, family discipline and image of the human being]. Revista de Psicología Social, 8(1), 83-97.

Ren, J., Hu, L., Zhang, H. \& Huang, Z. (2010). Implicit positive emotion counteracts ego depletion. Social Behavior and Personality, 38 (7), 919-928. http://dx.doi.org/10.2224/sbp. 2010.38.7.919

Retuerto, A.R. (2004). Diferencias en empatía en función de las variables género y edad [Differences in empathy function gender and age]. Apuntes de Psicología, 22(3), 323-339.

Reyna, C. \& Brussino, S. (2009). Propiedades psicométricas de la escala de comportamiento preescolar y jardín infantil en una muestra de niños argentinos de 3 a 7 años [Psychometric 
properties of the preschool and kindergarten behavior scales in a sample of Argentinean children from 3 to 7 years old]. Psykhe, 18(2), 127-140.

Richaud de Minzi, M.C. \& Oros, L. (2009). Emociones positivas, flexibilidad cognitiva y afrontamiento del estrés en niños [Positive emotions, cognitive flexibility and stress coping in children]. Trabajo presentado en el $32^{\circ}$ Congreso Interamericano de Psicología. Guatemala.

Richaud de Minzi, M.C., De Monte, G.L., Musso, M.F., Ábalos, C.C. \& Biberberg, C. (2005). La psicología positiva: Una nueva propuesta para la investigación psicológica [Positive Psychology: A new approach to psychological research]. Manuscrito no publicado. Facultad de Humanidades, UCA. Paraná, Argentina.

Richaud de Minzi, M.C., Lemos, V. \& Oros, L.B. (2013). Cuestionario Multidimensional de Empatía para Niños de 9 a 12 años [Multidimensional Empathy Questionnaire for Children 9 to 12 years]. Trabajo presentado en el XXXIV Congreso Interamericano de Psicología. Brasilia, Brasil.

Roche, R. (1982). Los orígenes de la conducta altruista en niños: Aspectos educativos y televisión en familia [The origins of altruistic behavior in children: Aspects of education and television at family]. Infancia y Aprendizaje, 19, 101-114.

Sanmartín, M.G., Carbonell, A.E. \& Baños, C.P. (2011). Relaciones entre empatía, conducta prosocial, agresividad, autoeficacia y responsabilidad personal y social de los escolares [Relationships among empathy, prosocial behavior, aggressiveness, self-efficacy and pupils' personal and social responsibility]. Psicothema, 23(1), 13-19.

Schulz, A. (2008). Validación de un sistema de evaluación de las habilidades sociales en niños argentinos por medio de informantes clave. Resultados preliminares [Validation of a system for assessing social skills in Argentinean children through key informant]. Informe final del proyecto de investigación PI 26/07. Paraná, Entre Ríos: Universidad Adventista del Plata.

Sroufe, L.A., Schork, E., Motti, F., Lawroski, N. \& LaFreniere, P. (1984). The role of affect in social competence. En C.E. Izard., J. Kagan \& R.B. Zajonc (Eds.), Emotions, cognition and behavior (pp. 289-319). Cambridge: Cambridge University Press.

Strümpfer, D. (2006). Positive emotions, positive emotionality and their contribution to fortigenic living: A review. South African Journal of Psychology, 36(1), 144-167. http://dx.doi. org/10.1177/008124630603600109

Wood, A.M., Maltby, J., Gillett, R., Linley, P.A. \& Joseph, S. (2008). The role of gratitude in the development of social support, stress, and depression: Two longitudinal studies. Journal of Research in Personality, 42, 854-871. http://dx.doi.org/10.1016/j.jrp.2007.11.003 
